\title{
STAT5B mutations in heterozygous state have negative impact on height: another clue in human stature heritability
}

\author{
Renata C Scalco, Vivian Hwa', Horacio M Domené2 ${ }^{2}$ Héctor G Jasper', \\ Alicia Belgorosky ${ }^{3}$, Roxana Marino ${ }^{3}$, Alberto M Pereira ${ }^{4}$, Carlos A Tonelli ${ }^{5}$, \\ Jan M Wit ${ }^{6}$, Ron G Rosenfeld ${ }^{7}$ and Alexander A L Jorge
}

Unidade de Endocrinologia Genetica, Laboratorio de Endocrinologia Celular e Molecular LIM/25, Disciplina de Endocrinologia da Faculdade de Medicina da Universidade de Sao Paulo, Avenida Dr Arnaldo, $4555^{\circ}$ Andar Sala 5340, 01246-903 Sao Paulo, Sao Paulo, Brazil, 'Division of Endocrinology, Cincinnati Center for Growth Disorders, Cincinnati Children's Hospital Medical Center, Cincinnati, Ohio 45229, USA, ${ }^{2}$ Centro de Investigaciones Endocrinológicas 'Dr César Bergadá' (CEDIE), CONICET - FEI - División de Endocrinología, Hospital de Niños Ricardo Gutiérrez, C1425EFD Buenos Aires, Argentina, ${ }^{3}$ Endocrine Service, Hospital de Pediatria Garrahan Ciudad Autonoma de Buenos Aires Pozos 1881, 1245 Buenos Aires, Argentina, ${ }^{4}$ Division of Endocrinology, Department of Medicine, Leiden University Medical Center, 2300 RC Leiden, The Netherlands, ${ }^{5}$ Universidade do Extremo Sul Catarinense, 88806-000 Criciúma, Santa Catarina, Brazil, ${ }^{6}$ Department of Pediatrics, Leiden University Medical Center, 2300 RC Leiden, The Netherlands and ${ }^{7}$ Department of Pediatrics, Oregon Health and Science University, Portland, Oregon 97239, USA

Correspondence should be addressed to A A L Jorge Email alexj@usp.br or alexj@pq.cnpq.br

\begin{abstract}
Context and objective: GH insensitivity with immune dysfunction caused by STAT5B mutations is an autosomal recessive condition. Heterozygous mutations in other genes involved in growth regulation were previously associated with a mild height reduction. Our objective was to assess for the first time the phenotype of heterozygous STAT5B mutations. Methods: We genotyped and performed clinical and laboratory evaluations in 52 relatives of two previously described Brazilian brothers with homozygous STAT5B c.424_427del mutation (21 heterozygous). Additionally, we obtained height data and genotype from 1104 adult control individuals from the same region in Brazil and identified five additional families harboring the same mutation (18 individuals, 11 heterozygous). Furthermore, we gathered the available height data from first-degree relatives of patients with homozygous STAT5B mutations (17 individuals from seven families). Data from heterozygous individuals and non-carriers were compared.

Results: Individuals carrying heterozygous STAT5B c.424_427del mutation were 0.6 SDS shorter than their non-carrier relatives $(P=0.009)$. Heterozygous subjects also had significantly lower SDS for serum concentrations of IGF1 $(P=0.028)$ and IGFBP3 $(P=0.02)$ than their non-carrier relatives. The 17 heterozygous first-degree relatives of patients carrying homozygous STAT5B mutations had an average height SDS of $-1.4 \pm 0.8$ when compared with population-matched controls $(P<0.001)$. Conclusions: STAT5B mutations in the heterozygous state have a significant negative impact on height $(\sim 3.9 \mathrm{~cm})$. This effect is milder than the effect seen in the homozygous state, with height usually within the normal range. Our results support the hypothesis that heterozygosity of rare pathogenic variants contributes to normal height heritability.
\end{abstract}

\section{Introduction}

Previous studies have demonstrated that while homozygous mutations in genes involved in growth regulation are the cause of severe syndromic short stature, heterozygosity of the same variants can be associated with a milder height reduction $(1,2,3,4)$. For instance, in the growth hormone (GH) insulin-like growth factor 1
(C) 2015 European Society of Endocrinology Printed in Great Britain
Published by Bioscientifica Ltd. 
(IGF1) axis, heterozygous carriers of mutations in acidlabile subunit gene (IGFALS) (3) and IGF1 gene (1) were shown to be significantly shorter than non-carriers, although generally still within the normal height range. These data support the concept that rare mono-allelic variants with moderate effects on phenotype can be associated with height variability (5) and, as such, can be an etiology for non-syndromic short stature $(6,7)$.

STAT5B is a key mediator of GH signaling, as well as of other signaling pathways, including those of prolactin and interleukin 2 (IL2) (8). Since 2003, ten patients have been reported to harbor seven different homozygous STAT5B mutations $(9,10,11,12,13,14,15,16)$. These rare homozygous mutations in STAT5B cause GH insensitivity (GHI) and manifestations of immune dysregulation, such as increased susceptibility for opportunistic infections, lymphoid interstitial pneumonia, and eczema. GHI syndrome, classically associated with homozygous mutations in the $\mathrm{GH}$ receptor gene $(G H R)$, is characterized by severe postnatal growth failure, normal to elevated $\mathrm{GH}$ levels, and low serum concentrations of ALS, IGF1, and IGF binding protein 3 (IGFBP3). Unlike most GHI patients carrying defects in $G H R$, however, serum concentrations of $\mathrm{GH}$ binding protein (GHBP), the proteolytically cleaved extracellular domain of GHR, were normal and prolactin levels were increased in patients carrying homozygous STAT5B mutations (reviewed in (8)).

To date, STAT5B deficiency is considered an autosomal recessive condition. The impact of heterozygous STAT5B mutations on growth and the GH-IGF axis, however, has not been carefully evaluated, due in part to the rarity of described cases and families. To address this issue, we evaluated a large community in which multiple members carry a previously described STAT5B frameshift mutation (15). By comparing their data with data from other families harboring other mutations in $S T A T 5 B$, we provide evidence that heterozygous $S T A T 5 B$ mutations can influence stature.

\section{Subjects and methods}

\section{Subjects}

We evaluated 52 relatives of two Brazilian brothers with characterized GHI due to homozygous STAT5B c.424_427del mutation. Furthermore, an active search was done to investigate the prevalence of this mutation in the region where the index cases were born, identifying five unrelated heterozygous individuals among 1104 evaluated adult control subjects. Relatives of these five individuals were subsequently evaluated, totaling
18 subjects. Height data gathered from the remaining 1099 adult control individuals (non-carriers of STAT5B c.424_427del mutation) in the same region were used to assess the local population height.

Additionally, we gathered the available height data from first-degree heterozygous relatives of previously reported patients with homozygous STAT5B mutations. We also included in this group two recently diagnosed individuals heterozygous for STAT5B c.424_427del mutation, who lost two children with the same phenotype seen in patients with homozygous STAT5B mutations. In total, height data from 17 first-degree relatives from seven families were analyzed.

These studies were approved by the local ethics committees, and the patients or guardians gave their written informed consent.

\section{Genotyping in families with STAT5B c.424_427del mutation}

Genomic DNA was isolated from peripheral blood leukocytes using standard techniques. Genotyping for STAT5B c.424_427del mutation was done by fragment analysis technique. The primers were designed to amplify the region around this mutation (primer sequences and amplification protocols are available upon request). Genotyping was performed after the clinical evaluation.

\section{Clinical and laboratory assessment in families with STAT5B c.424_427del mutation}

Individuals from families with STAT5B c.424-427del mutation were evaluated by an investigator blinded for STAT5B genotype. They were questioned about pneumopathies, eczema, and other immune dysfunctions. Height and weight were assessed in all individuals. Total blood count, fasting glucose, and insulin, IgG, immunoglobulin A, and immunoglobulin E, basal GH, IGF1, IGFBP3, and prolactin were tested in $91 \%$ of the evaluated individuals. Serum GH, IGF1, IGFBP3, prolactin, and immunoglobulin E were measured through chemiluminescence assays and immunoglobulin A and IgG through turbidimetry. IGF1 and IGFBP3 were transformed to SDS (17).

\section{Whole-exome sequencing}

Whole-exome sequencing of genomic DNA, obtained from the peripheral blood of one individual heterozygous for STAT5B c.424_427del mutation and with pneumopathy of an unknown etiology, was performed with Illumina's Nextera Exome Enrichment kits (Illumina, San Diego, 
CA, USA) for library preparation and exome capture and the Illumina HiSeq sequencer. Alignments and variant annotation were made as previouly described (18).

\section{Statistical methods}

Because the patients came from many ethnic groups, height data were expressed as SDS for the appropriate country/ethnic group. The effect of one mutant allele vs WT was determined in the whole group.

Groups were compared by unpaired $t$-test or ANOVA followed by Tukey test for numerical variables with normal distribution. Numerical variables without parametric distribution were analyzed by Mann-Whitney Rank Sum Test or Kruskal-Wallis ANOVA on Ranks. Categorical data were compared between groups through $\chi^{2}$ test or Fisher's exact test as appropriate. Statistical significance was assumed for $P<0.05$. Statistical analysis was made with SigmaStat 3.5 (Systat Software, Inc., Chicago, IL, USA) and MedCalc version 11.1.1.0 (MedCalc Software, Mariakerke, Belgium).

\section{Results}

\section{Families harboring STAT5B c.424_427del mutation}

The largest Brazilian family consisted of two patients with homozygous STAT5B c.424_427del mutation, 21 heterozygous carriers (including their non-consanguineous parents), and 31 non-carrier relatives (Supplementary
Figure 1, see section on supplementary data given at the end of this article). The other five families identified consisted of 11 heterozygous carriers and seven non-carrier relatives. When polymorphic markers around this mutation were studied, the same haplotype was found in these six families, which was consistent with the presence of a founder effect (data not shown) (19). Consequently, we analyzed all individuals from the six families together (Table 1).

In total, we analyzed data from 32 heterozygous carriers of STAT5B c.424_427del mutation (17 males) and 38 non-carrier family members (12 males). Unrelated spouses were not included. One heterozygous carrier was excluded from the height analysis because of severe short stature (height SDS -3.5) of unknown cause. Among the 70 evaluated individuals (aged $32.7 \pm 18.5$ years old), 16 were children (seven heterozygous for STAT5B mutation).

Non-carrier subjects in these families had a similar height SDS to individuals from the local population (height SDS $-0.2 \pm 1.0$ vs $-0.4 \pm 0.8$ respectively, $P=0.63$ ) Heterozygous STAT5B c.424_427del individuals were significantly shorter (height SDS $-0.8 \pm 0.9$ ) than their non-carrier relatives (height SDS difference of -0.6 , $P=0.009$, CI 95\% -1.1 to -0.2 ), although all were within the normal height range (Table 1). When the analysis was done excluding the children, the same results were obtained (height SDS $-0.8 \pm 0.9$ vs $-0.2 \pm 1.0$ for heterozygous and non-carrier relatives respectively, $P=0.02$, Fig. 1 ). Furthermore, heterozygous carriers had significantly lower IGF1 and IGFBP3 SDS than their non-carrier relatives (Table 1).

Table 1 Clinical and biochemical characteristics of heterozygous carriers of STAT5B c.424_427del mutation vs non-carriers. Values are expressed as mean \pm s.D.

\begin{tabular}{|c|}
\hline $\begin{array}{l}n \\
\text { Dermopathy } \\
\text { Severe pneumopathy } \\
\text { Height SDS } \\
\text { Basal GH (ng/ml) } \\
\text { IGF1 SDS } \\
\text { IGFBP3 SDS } \\
\text { Prolactin }(\mathrm{ng} / \mathrm{ml}) \\
\text { Glucose }(\mathrm{mg} / \mathrm{dl}) \\
\text { Insulin }(\mu U \mathrm{Ul} / \mathrm{ml}) \\
\text { Hemoglobin }(\mathrm{g} / \mathrm{dl}) \\
\text { Leucocytes }\left(\mathrm{cell} / \mathrm{mm}^{3}\right) \\
\text { Lymphocytes }\left(\mathrm{cells} / \mathrm{mm}^{3}\right) \\
\text { IgG (mg/dl) } \\
\text { IgA (mg/dl) } \\
\text { IgE (Ul/ml) }\end{array}$ \\
\hline
\end{tabular}

\begin{tabular}{c}
\hline WT/Mut \\
\hline 32 \\
$9: 32$ \\
$1: 32$ \\
$-0.8 \pm 0.9^{a}$ \\
$1.4 \pm 2.2$ \\
$-0.4 \pm 1.2$ \\
$-0.9 \pm 1.4$ \\
$12.5 \pm 7.2$ \\
$90 \pm 14$ \\
$6.9 \pm 7.3$ \\
$13.3 \pm 1.3$ \\
$6893 \pm 2008$ \\
$3998 \pm 1292$ \\
$1022 \pm 205$ \\
$244 \pm 141$ \\
$220 \pm 325$
\end{tabular}

\begin{tabular}{c}
\hline $\mathbf{W T / W T}$ \\
\hline 38 \\
$1: 38$ \\
$0: 38$ \\
$-0.2 \pm 1.0$ \\
$1.0 \pm 1.6$ \\
$0.3 \pm 1.2$ \\
$0.0 \pm 1.5$ \\
$13.0 \pm 10.6$ \\
$83 \pm 13$ \\
$6.4 \pm 3.9$ \\
$13.4 \pm 1.5$ \\
$6935 \pm 2695$ \\
$3598 \pm 1329$ \\
$1054 \pm 249$ \\
$225 \pm 123$ \\
$143 \pm 191$
\end{tabular}

\begin{tabular}{cc}
\hline $\boldsymbol{P}$ \\
\hline 0.004 \\
NS \\
0.009 \\
NS \\
0.028 \\
0.02 \\
NS \\
NS \\
NS \\
NS \\
NS \\
NS \\
NS \\
NS \\
NS
\end{tabular}

$\mathrm{GH}$, growth hormone; IGF1, insulin-like growth factor 1; IGFBP3, insulin-like growth factor binding protein 3; IgA/lgE, immunoglobulins A and E; NS, non-significant.

${ }^{a}$ Excluding one heterozygous carrier with severe short stature (height SDS - 3.5). 


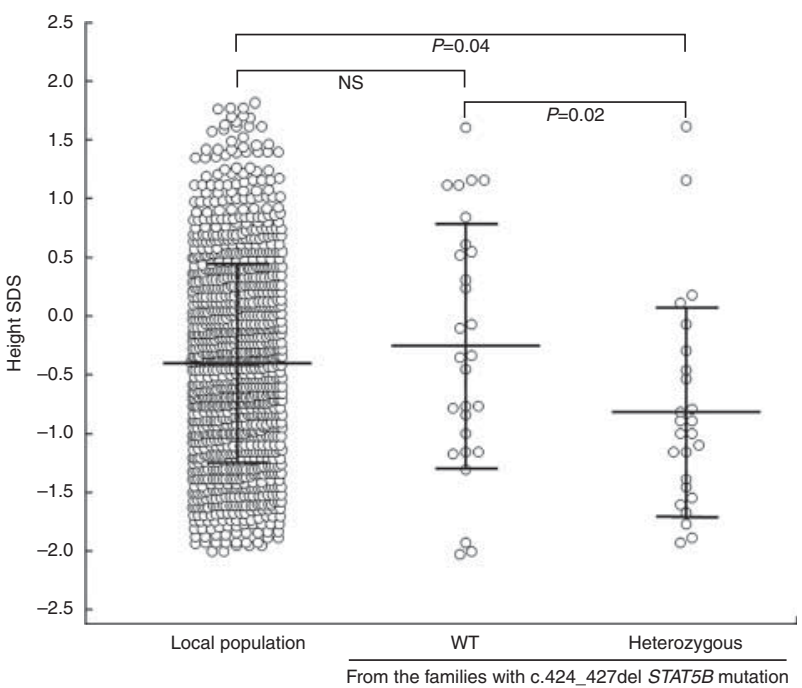

\section{Figure 1}

Comparison of height SDS distribution among adult heterozygous carriers of STAT5B c.424_427del mutation $(n=25)$, their non-carrier relatives $(n=28)$, and a population sample from the same region in the south of Brazil $(n=1099)$.

Other parameters, such as basal GH and prolactin concentrations, were not different between these groups.

Present or past history of dermopathies was reported in nine out of 32 individuals heterozygous for STAT5B c.424_427del mutation and in one out of 38 individuals who were non-carriers $(P=0.004)$. We clinically diagnosed eczema in four carriers. No differences in total blood count and immunoglobulin levels between carriers and noncarriers were observed (Table 1).

One cousin of the probands, who was heterozygous for STAT5B c.424_427del mutation, presented with a moderate to severe restrictive pneumopathy of unknown etiology. Her disease was milder than the pneumopathy observed in patients homozygous for STAT5B mutations, since she was in her thirties and still not oxygen dependent. Exome sequencing excluded other STAT5B mutations and mutations in genes normally associated with pneumopathies (data not shown). A lung biopsy of this patient showed areas of interstitial thickening near respiratory bronchioles, inflammatory interstitial infiltrate with lymphocytes, plasmocytes and histiocytes, and mild interstitial fibrosis, which is compatible with lymphoid interstitial pneumonia. Her father, an obligatory heterozygous carrier for the same mutation, died of respiratory failure secondary to an uninvestigated chronic pneumopathy.

\section{First-degree relatives of index cases carrying STAT5B mutations}

Height data were obtained in 17 first-degree relatives of ten patients homozygous for STAT5B mutations (Table 2). Two Argentinian patients were adopted soon after birth and, consequently, data from their biological relatives were not available. Parents were consanguineous in four families and not consanguineous in three families. All of these relatives were heterozygous for STAT5B mutations with an average height SDS of $-1.4 \pm 0.8$ when compared with appropriate population-matched controls $(P<0.001)$.

\section{Discussion}

In adequate health and nutritional conditions, genetic variation is the main determinant of stature, accounting for $\sim 80 \%$ of height variability (20). Recent genome-wide association studies (GWAS) identified 697 variants in

Table 2 Data of index patients homozygous for STAT5B mutations and their first-degree relatives.

\begin{tabular}{|c|c|c|c|c|c|c|c|c|}
\hline \multirow[b]{2}{*}{ Family no. } & \multirow[b]{2}{*}{ Reference } & \multirow[b]{2}{*}{ Consanguinity } & \multirow{2}{*}{$\begin{array}{c}\text { CDNA } \\
\text { mutation }\end{array}$} & \multirow[b]{2}{*}{ Origin } & \multicolumn{4}{|c|}{ Local height SDS } \\
\hline & & & & & Patient & Fathers & Mothers & Siblings \\
\hline 1 & (9) & Yes & C. $1888 \mathrm{G}>\mathrm{C}$ & Argentina & -7.5 & -0.3 & -1.2 & \\
\hline 2 & (10) & Yes & c.1191insG & Turkey & -7.8 & -0.9 & -0.6 & \\
\hline 3 & (11) & No & c.1102insC & Caribbean & -5.9 & -2.8 & -0.8 & $-2.3 /-0.8$ \\
\hline 4 & $(12)$ & No & c. $454 C>T$ & Argentina & -9.9 & -2.2 & -3.3 & -2.0 \\
\hline 5 & (13) & Yes & c. 1680 delG & Kuwait & $-5.6 /-5.8$ & -1.3 & -0.6 & \\
\hline 6 & (14) & Adopted & c. $454 C>T$ & Argentina & -5.3 & NA & NA & \\
\hline 7 & (15) & No & c.424_427del & Brazil & $-5.6 /-3.0$ & -1.5 & -1.0 & \\
\hline 8 & (16) & Adopted & c. $1937 \overline{7}>C$ & Argentina & -5.95 & NA & NA & \\
\hline 9 & (19) & Yes & c.424_427del & Brazil & $\mathrm{NA}^{\mathrm{a}}$ & -0.9 & -1.9 & \\
\hline Mean $\pm S D S$ & & & & & $-6.2 \pm 1.8$ & & $-1.4 \pm 0.8$ & \\
\hline Median (range) & & & & & $-5.9(-9.9 ;-3.0)$ & & $-1.2(-3.3 ;-0.3)$ & \\
\hline
\end{tabular}


423 loci that, together, accounted for only one-fifth of adult height heritability (21). The individual effect of single nucleotide polymorphisms (SNPs) found in these studies, furthermore, is very small $(<0.5 \mathrm{~cm})(22)$. The inability of GWAS to explain all height heritability, despite the increasing number of evaluated individuals, suggests that numerous rare variants with a largeto-moderate effect have a role in height variability (23). However, it is difficult to evaluate the importance of rare variants in height variability through the current available methods, since each private allele is restricted to a few families or small populations.

In the present study, the analysis of a large family with many heterozygous carriers of the STAT5B c.424_427del mutation showed that these individuals are significantly shorter than their non-carrier relatives and local population controls (mean height SDS difference of 0.6). Assuming that the mean s.D. for adult height distribution is $6.5 \mathrm{~cm}$, the mean height loss seen in these individuals can be estimated at $3.9 \mathrm{~cm}$, which is a much larger individual effect than the $0.5 \mathrm{~cm}$ attributed to SNPs identified in GWAS. The significant reduction in IGF1 SDS and IGFBP3 SDS seen with STAT5B c.424_427del heterozygous carriers, furthermore, suggests that a decreased responsiveness to $\mathrm{GH}$ action could explain, at least in part, the observed height reduction.

Moreover, the analysis of the available height data from carriers of the different STAT5B mutations also displayed a significant reduction in height when compared to their population controls and was equivalent to a height decrease of $9.1 \mathrm{~cm}$, an even greater difference than that observed for carriers of STAT5B c.424_427del mutation. This difference could be due to the relatively smaller number of first-degree relatives available for study and/or to the variable effects on height dependent on the individual STAT5B mutation itself. For the STAT5B c.424_427del mutation, the lack of expression of the mutant protein in reconstitution experiments ( $\mathrm{V}$ Hwa, unpublished data) suggests that partial haploinsufficiency could explain the modest height reduction seen in heterozygous carriers. No dominant-negative STAT5B mutations have been reported to date, although, interestingly, a heterozygous STAT5Bp.Gln177Pro variant was recently described in two GHI patients with severe short stature but no immunological dysfunction (24).

Heterozygous mutations in other genes along the GHIGF1 axis similarly show larger individual effects on height than SNPs, supporting our finding. For example, in a family carrying IGF1 p.V44M mutation, individuals heterozygous for this mutation were 0.6 SDS shorter (equivalent to $3.9 \mathrm{~cm}$ ) than their non-carrier relatives (1). Moreover, heterozygous carriers of IGFALS mutations were 0.9 SDS shorter (equivalent to $5.8 \mathrm{~cm}$ ) than their non-carrier relatives (3). Heterozygous mutations in genes associated with bone growth regulation similarly impacted height: in a large family with many individuals heterozygous for a NPR2 mutation, carriers were 1.4 SDS shorter than noncarriers (equivalent to $9.1 \mathrm{~cm}$ ) (2). In all of these studies, the clinical presentation of heterozygous individuals was much milder than the disorder seen in patients homozygous for the same mutations. Altogether, the presence of these rare pathogenic mutations in the heterozygous state suggests that the loss of one functional allele may result in lownormal height and borderline short stature.

Finally, we observed that individuals with heterozygous STAT5B c.424_427del mutation reported more dermopathies and skin allergies when compared to their non-carrier relatives $(P=0.004)$. No difference in pneumopathies or other allergies was reported by both groups, although two heterozygous carriers (a cousin of the probands and her father) had severe pneumopathy of unknown etiology. Further investigations are necessary to better characterize the potential effects of heterozygous STAT5B mutations in immunologic alterations.

In conclusion, we demonstrated that STAT5B mutations in the heterozygous state exert a significant negative impact on height. This effect is milder than the effect seen in the homozygous state, with height usually within the low normal range. Our results support the hypothesis that heterozygosity of rare pathogenic variants contributes to normal height heritability. Whether the cumulative effect of such variants could be responsible for a proportion of the missing height heritability posed by GWAS studies remains to be determined.

\section{Supplementary data}

This is linked to the online version of the paper at http://dx.doi.org/10.1530/ EJE-15-0398.

Declaration of interest

The authors declare that there is no conflict of interest that could be perceived as prejudicing the impartiality of the research reported.

\section{Funding}

This work was supported by Grants 2013/03236-5, 2010/19809-6 (to A A L Jorge), and 2011/15078-0 (to R C Scalco) from the Sao Paulo Research Foundation (FAPESP); Grant 304678/2012-0 (to A A L Jorge) from the National Council for Scientific and Technological Development (CNPq) and NIH R01HD078592 (to V Hwa). 


\section{Acknowledgements}

The authors thank Dr Julio Cezar Cechinel from Laboratorio Pasteur, Criciúma, SC, Brazil, for performing laboratory tests in individuals from families with STAT5B C.424_427del mutation.

\section{References}

1 Walenkamp MJ, Karperien M, Pereira AM, Hilhorst-Hofstee Y, van Doorn J, Chen JW, Mohan S, Denley A, Forbes B, van Duyvenvoorde HA et al. Homozygous and heterozygous expression of a novel insulin-like growth factor-I mutation. Journal of Clinical Endocrinology and Metabolism 200590 2855-2864. (doi:10.1210/jc.2004-1254)

2 Olney RC, Bukulmez H, Bartels CF, Prickett TC, Espiner EA, Potter LR \& Warman ML. Heterozygous mutations in natriuretic peptide receptor-B (NPR2) are associated with short stature. Journal of Clinical Endocrinology and Metabolism 200691 1229-1232. (doi:10.1210/jc.2005-1949)

3 Fofanova-Gambetti OV, Hwa V, Wit JM, Domene HM, Argente J, Bang P, Hogler W, Kirsch S, Pihoker C, Chiu HK et al. Impact of heterozygosity for acid-labile subunit (IGFALS) gene mutations on stature: results from the international acid-labile subunit consortium. Journal of Clinical Endocrinology and Metabolism 201095 4184-4191. (doi:10.1210/jc.2010-0489)

4 Jorge AA, Funari MF, Nishi MY \& Mendonca BB. Short stature caused by isolated SHOX gene haploinsufficiency: update on the diagnosis and treatment. Pediatric Endocrinology Reviews: PER 20108 79-85.

5 Gibson G. Rare and common variants: twenty arguments. Nature Reviews. Genetics 201213 135-145. (doi:10.1038/nrg3118)

6 Malaquias AC, Scalco RC, Fontenele EG, Costalonga EF, Baldin AD, Braz AF, Funari MF, Nishi MY, Guerra-Junior G, Mendonca BB et al. The sitting height/height ratio for age in healthy and short individuals and its potential role in selecting short children for SHOX analysis. Hormone Research in Poediatrics 201380 449-456. (doi:10.1159/000355411)

7 Vasques GA, Arnhold IJ \& Jorge AA. Role of the natriuretic peptide system in normal growth and growth disorders. Hormone Research in Poediatrics 201482 222-229. (doi:10.1159/000365049)

8 Hwa V, Nadeau K, Wit JM \& Rosenfeld RG. STAT5b deficiency: lessons from STAT5b gene mutations. Best Practice \& Research. Clinical Endocrinology \& Metabolism 201125 61-75. (doi:10.1016/j.beem.2010.09.003)

9 Kofoed EM, Hwa V, Little B, Woods KA, Buckway CK, Tsubaki J, Pratt KL, Bezrodnik L, Jasper H, Tepper A et al. Growth hormone insensitivity associated with a STAT5b mutation. New England Journal of Medicine 2003349 1139-1147. (doi:10.1056/NEJMoa022926)

10 Hwa V, Little B, Adiyaman P, Kofoed EM, Pratt KL, Ocal G, Berberoglu M \& Rosenfeld RG. Severe growth hormone insensitivity resulting from total absence of signal transducer and activator of transcription 5b. Journal of Clinical Endocrinology and Metabolism 2005 90 4260-4266. (doi:10.1210/jc.2005-0515)

11 Vidarsdottir S, Walenkamp MJ, Pereira AM, Karperien M, van Doorn J, van Duyvenvoorde HA, White S, Breuning MH, Roelfsema F, Kruithof MF et al. Clinical and biochemical characteristics of a male patient with a novel homozygous STAT5b mutation. Journal of Clinical Endocrinology and Metabolism 200691 3482-3485. (doi:10.1210/jc.2006-0368)

12 Bernasconi A, Marino R, Ribas A, Rossi J, Ciaccio M, Oleastro M, Ornani A, Paz R, Rivarola MA, Zelazko M et al. Characterization of immunodeficiency in a patient with growth hormone insensitivity secondary to a novel STAT5b gene mutation. Pediatrics $2006 \mathbf{1 1 8}$ e1584-e1592. (doi:10.1542/peds.2005-2882)

13 Hwa V, Camacho-Hubner C, Little BM, David A, Metherell LA, El-Khatib N, Savage MO \& Rosenfeld RG. Growth hormone insensitivity and severe short stature in siblings: a novel mutation at the exon 13-intron 13 junction of the STAT5b gene. Hormone Research $2007 \mathbf{6 8}$ 218-224. (doi:10.1159/000101334)

14 Boyanovsky A, Lozano A \& Testa G. Growth hormone insensitivity and immunodeficiency: mutation in the STAT5B gene. In 8th Joint Meeting of the Lawson Wilkins Pediatric Endocrine Society/European Society for Paediatric Endocrinology pp P01-P67, 2009.

15 Pugliese-Pires PN, Tonelli CA, Dora JM, Silva PC, Czepielewski M, Simoni G, Arnhold IJ \& Jorge AA. A novel STAT5B mutation causing GH insensitivity syndrome associated with hyperprolactinemia and immune dysfunction in two male siblings. European Journal of Endocrinology/European Federation of Endocrine Societies 2010163 349-355. (doi:10.1530/EJE-10-0272)

16 Scaglia PA, Martinez AS, Feigerlova E, Bezrodnik L, Gaillard MI, Di Giovanni D, Ballerini MG, Jasper HG, Heinrich JJ, Fang P et al. A novel missense mutation in the $\mathrm{SH} 2$ domain of the STAT5B gene results in a transcriptionally inactive STAT5b associated with severe IGF-I deficiency, immune dysfunction, and lack of pulmonary disease. Journal of Clinical Endocrinology and Metabolism 201297 E830-E839. (doi:10.1210/jc.2011-2554)

17 Elmlinger MW, Kuhnel W, Weber MM \& Ranke MB. Reference ranges for two automated chemiluminescent assays for serum insulin-like growth factor I (IGF-I) and IGF-binding protein 3 (IGFBP-3). Clinical Chemistry and Laboratory Medicine: CCLM/FESCC 200442 654-664. (doi:10.1515/CCLM.2004.112)

18 Bertola DR, Yamamoto GL, Almeida TF, Buscarilli M, Jorge AA, Malaquias AC, Kim CA, Takahashi VN, Passos-Bueno MR \& Pereira AC. Further evidence of the importance of RIT1 in Noonan syndrome. American Journal of Medical Genetics. Part A 2014 164A 2952-2957. (doi:10.1002/ajmg.a.36722)

19 Scalco RC, Funari MF, Aracava RM, Tonelli CA \& Jorge AA. Evidence for a Founder Effect of C.424_427del STAT5B Mutation Causing Growth Hormone Insensitivity in the South of Brazil. In Endocrine Society's 96th Annual Meeting and Expo, pp MON-0161. Ed E Society. Chicago: Endocrine Review, 2014.

20 Silventoinen K, Sammalisto S, Perola M, Boomsma DI, Cornes BK, Davis C, Dunkel L, De Lange M, Harris JR, Hjelmborg JV et al. Heritability of adult body height: a comparative study of twin cohorts in eight countries. Twin Research 20036 399-408. (doi:10.1375/136905203770326402)

21 Wood AR, Esko T, Yang J, Vedantam S, Pers TH, Gustafsson S, Chu AY, Estrada K, Luan J, Kutalik Z et al. Defining the role of common variation in the genomic and biological architecture of adult human height. Nature Genetics 201446 1173-1186. (doi:10.1038/ng.3097)

22 Lettre G. Genetic regulation of adult stature. Current Opinion in Pediatrics 200921 515-522. (doi:10.1097/MOP.0b013e32832c6dce)

23 Johansson A, Jonasson I \& Gyllensten U. Extended haplotypes in the growth hormone releasing hormone receptor gene (GHRHR) are associated with normal variation in height. PLOS ONE 20094 e4464. (doi:10.1371/journal.pone.0004464)

24 Klammt J, Neumann D, Andrew SF, Vokurkova D, Stobbe H, Buckham K, Rosenfeld RG, Pfäffle R \& Hwa V. Severe Short Stature and GH Insensitivity Due to a De Novo Heterozygous STAT5B Missense Mutation. In Endocrine Society's 96th Annual Meeting and Expo, pp OR24-23. Chicago: Endocrine Society, 2014.

Received 15 April 2015

Revised version received 22 May 2015

Accepted 1 June 2015 